\title{
Effects of autozygosity and schizophrenia polygenic risk on cognitive and brain developmental trajectories
}

\author{
Aldo Córdova-Palomera ${ }^{1}$ Tobias Kaufmann ${ }^{1} \cdot$ Francesco Bettella $\mathbb{D}^{1}{ }^{1}$ Y Yunpeng Wang $\mathbb{D}^{1} \cdot$ Nhat Trung Doan ${ }^{1}$. \\ Dennis van der Meer ${ }^{1} \cdot$ Dag Alnæs $^{1} \cdot$ Jaroslav Rokicki $^{1,2} \cdot$ Torgeir Moberget $^{1} \cdot$ Ida Elken Sønderby $^{1} \cdot$ \\ Ole A. Andreassen ${ }^{1} \cdot$ Lars T. Westlye ${ }^{1,2}$
}

Received: 20 June 2017 / Revised: 28 February 2018 / Accepted: 2 March 2018 / Published online: 27 April 2018

(c) European Society of Human Genetics 2018

\begin{abstract}
Cognitive and brain development are determined by dynamic interactions between genes and environment across the lifespan. Aside from marker-by-marker analyses of polymorphisms, biologically meaningful features of the whole genome (derived from the combined effect of individual markers) have been postulated to inform on human phenotypes including cognitive traits and their underlying biological substrate. Here, estimates of inbreeding and genetic susceptibility for schizophrenia calculated from genome-wide data-runs of homozygosity $(\mathrm{ROH})$ and schizophrenia polygenic risk score (PGRS) — are analyzed in relation to cognitive abilities $(n=4183)$ and brain structure $(n=516)$ in a general-population sample of European-ancestry participants aged 8-22, from the Philadelphia Neurodevelopmental Cohort. The findings suggest that a higher ROH burden and higher schizophrenia PGRS are associated with higher intelligence. Cognition-ROH and cognition-PGRS associations obtained in this cohort may, respectively, evidence that assortative mating influences intelligence, and that individuals with high schizophrenia genetic risk who do not transition to disease status are cognitively resilient. Neuroanatomical data showed that the effects of schizophrenia PGRS on cognition could be modulated by brain structure, although larger imaging datasets are needed to accurately disentangle the underlying neural mechanisms linking IQ with both inbreeding and the genetic burden for schizophrenia.
\end{abstract}

\section{Introduction}

Cognitive abilities and neural development are determined by complex and dynamic interactions between environmental influences and the individual genetic architecture across the lifespan $[1,2]$. The genetically driven temporal regulation is especially noticeable in the transition from childhood to

Electronic supplementary material The online version of this article (https://doi.org/10.1038/s41431-018-0134-2) contains supplementary material, which is available to authorized users.

$\triangle$ Aldo Córdova-Palomera

aldoc@medisin.uio.no

$\triangle$ Lars T. Westlye

1.t.westlye@psykologi.uio.no

1 NORMENT, KG Jebsen Centre for Psychosis Research, Division of Mental Health and Addiction, Oslo University Hospital \& Institute of Clinical Medicine, University of Oslo, Oslo, Norway

2 Department of Psychology, University of Oslo, Oslo, Norway adolescence and adulthood, and can have both immediate and lagged effects on the risk for psychiatric disorders depending on the timing of gene expression and the relevant environmental perturbations, which jointly determine the individual age of onset of the clinical phenotypes [3]. Studies on the genetic architecture of cognitive and structural brain phenotypes are eliciting previous findings from quantitative genetics (i.e., heritability) [4, 5]. However, a large fraction of the underlying molecular genetic mechanisms - and their relation to developmental trajectories-remains to be revealed.

Previous research suggests that runs of homozygosity $(\mathrm{ROH})$ are linked to human cognitive abilities [6-8]. Studies on $\mathrm{ROH}$ and cognition in different strata of the general population have shown mixed results, with moderate effect sizes [6-8]. While two studies [6, 8] suggest that inbreeding depression may decrease adult intelligence, divergent results by Power et al. [7] could be explained in view of three evidences. First, assortative mating has recently been highlighted as an important factor underlying psychiatric and behavioral phenotypes [9-12], in line with specific findings showing assortative mating in relation to cognitive ability 
published in the late 20th century [13, 14]. Second, similar to the age-varying pattern for the heritability of cognition [15, 16], the genetic effect of ROH on cognition may be different across the lifespan. Third, differences in ROH burden calculation between the report by Joshi et al. [6] and the other two studies [7, 8] may have added to the discrepancies.

Growing evidence indicates that the genetic architectures of schizophrenia and cognition are partly shared in young and middle-aged adults [17], and that the shared genetic variance between cognition and schizophrenia risk might be age-dependent [18], calling for further research on lifespan patterns. In fact, Germine et al. [19] reported that higher schizophrenia PGRS is linked to lower speed of emotion identification and speed of verbal reasoning in healthy young individuals from the Philadelphia Neurodevelopmental Cohort (PNC). Consistently, Shafee et al. [20] recently found an association between schizophrenia PGRS and specific cognitive abilities in adults. The latter findings may be mediated by genetic effects on brain structure and function, but the evidence of associations between genetic risk for schizophrenia and brain features is scarce, with some studies reporting no overlap between schizophrenia PGRS and subcortical phenotypes derived from magnetic resonance imaging (MRI) [21], whereas others suggest an association with cortical thickness/surface area [22]. Replication studies using independent samples and probably a wider span of brain features are thus needed.

With this background, using a sample of 4183 young participants (ages 8-22) of European ancestry from the PNC, we pursue the following four main aims. First, the putative links between multivariate genome-wide features $(\mathrm{ROH}$ burden and schizophrenia PGRS, computed independently) and both intellectual abilities and neuroanatomical features are tested. Second, potential age-modulating effects on relevant outcomes of the latter analysis are tested. Third, whether inbreeding modifies the effects of genome-wide schizophrenia burden on cognition and brain anatomy is evaluated by testing the effects of the interaction between ROH and PGRS on cognitive ability and brain structure. Finally, the presence of brain anatomy differences mediating the associations between $\mathrm{ROH} / \mathrm{PGRS}$ and cognition is assessed from a causal mediation framework. Using those elements, our overall goal is to outline potential neurodevelopmental pathways leading from biologically meaningful whole-genome features to neural and ultimately cognitive disruptions.

\section{Materials and methods}

\section{Participants and measures}

The data was retrieved from the PNC public domain resources. The PNC includes more than 9000 individuals aged 8-22 years drawn from a larger population, enrolled through a joint collaborative effort of the Center for Applied Genomics at Children's Hospital of Philadelphia and the Brain Behavior Laboratory (University of Pennsylvania). All enrolled participants were able to provide informed consent, and parental consent was also required for subjects under age 18. All individuals underwent a psychiatric evaluation using a structured clinical interview, and the Wide Range Achievement Test (WRAT) [23] was applied. After removing participants of nonEuropean ancestry or due to missing data for relevant variables, 4183 individuals were included in the main analyses (ROH, PGRS, and cognition), and MRI data was available for 516 of them. A more comprehensive description of the PNC demographics and data collection protocols is provided in previous publications [24-26]. PNC data is publicly available via dbGaP (https://www. ncbi.nlm.nih.gov/projects/gap/cgi-bin/study.cgi?study_ $\mathrm{id}=$ phs000607.v1.p1) and related resources (https://www. med.upenn.edu/bbl/philadelphianeurodevelopmenta lcohort.html). Analysis pipelines used in this study can be obtained upon request to the authors.

\section{Genotyping platforms and genotype imputation}

Genotyping was performed by the Center for Applied Genomics, at the Children's Hospital of Philadelphia. The DNA samples from the PNC were genotyped in different batches using the following platforms: Illumina OmniExpress $(n=1657)$, Illumina Human-610 Quad ( $n$ =3807), Illumina HumanHap-550-v1 $(n=556)$, Illumina HumanHap-550-v3 $(n=1914)$, Affymetrix GenomeWide Human SNP Array $6.0(n=66)$ or Affymetrix Axiom $(n=722)$ (hereafter, Omni, Quad, 550-v1, 550-v3, Affy60, and Axiom). From those datasets, only participants of European ancestry were included in the final statistical analyses, in recognition that the inclusion of subjects from other ethnicities might add genetic variability altering both ROH-phenotype and PGRS-phenotype association estimates (e.g., homozygosity might differ between ethnicities and across samples with distinct admixture levels [8], and schizophrenia PGRS might not explain much variance in non-European samples [27]). Further details on genotype imputation can be found in Supplementary Material.

\section{Runs of homozygosity}

The sum of the total length of ROHs across chromosomes 1-22 was divided by the total SNP-mappable autosomal length $\left(2.77 \times 10^{-9} \mathrm{bp}\right)$, to obtain an estimate of ROH burden for each individual ( $\mathrm{ROH}$ fraction, $F_{\mathrm{ROH}}$ ). The protocol was based on parameters widely used in previously published 
Table 1 Descriptive values of the main phenotypic and genetic variables included in the analyses, stratified by genotyping platform

\begin{tabular}{|c|c|c|c|c|c|}
\hline & Omni & Quad & $550-\mathrm{v} 1$ & $550-\mathrm{v} 3$ & $\begin{array}{l}\text { Between-group } \\
\text { comparison statistic } \\
(p \text {-value })^{\mathrm{d}}\end{array}$ \\
\hline \multicolumn{6}{|l|}{ Subjects $(n)$} \\
\hline Male/female & $573 / 590$ & $886 / 896$ & $148 / 154$ & $462 / 474$ & $0.094(0.993)$ \\
\hline \multicolumn{6}{|l|}{ Medical status } \\
\hline $0 / 1 / 2 / 3 / 4$ & $207 / 321 / 342 / 213 / 78$ & $264 / 529 / 496 / 368 / 112$ & $30 / 56 / 105 / 81 / 30$ & $103 / 220 / 337 / 216 / 49$ & $72.804\left(10^{-10}\right)$ \\
\hline \multicolumn{6}{|c|}{ Age at $\mathrm{CNB}^{\mathrm{a}}$ (years) } \\
\hline Median & 13 & 14 & 16 & 15 & $30.922\left(<3 \times 10^{-16}\right)$ \\
\hline Range & $8-21$ & $8-21$ & $8-22$ & $8-21$ & \\
\hline \multicolumn{6}{|c|}{ Raw WRAT score } \\
\hline Median & 54 & 55 & 57 & 55 & $7.091\left(9.5 \times 10^{-5}\right)$ \\
\hline Range & $18-70$ & $16-70$ & $24-69$ & $11-70$ & \\
\hline \multicolumn{6}{|c|}{ Standardized WRAT score (IQ, standard units) } \\
\hline Median & 106 & 105 & 106 & 105 & $4.106(0.006)$ \\
\hline Range & $64-145$ & $55-145$ & $71-145$ & $55-145$ & \\
\hline \multicolumn{6}{|c|}{ Intracranial volume $\left(10^{6} \mathrm{~mm}^{3}\right)^{\mathrm{b}}$} \\
\hline Median & 1.609 & 1.603 & 1.55 & 1.573 & $2.292(0.077)$ \\
\hline Range & $1.218-1.988$ & $1.095-1.98$ & $1.253-1.893$ & $1.179-1.849$ & \\
\hline \multicolumn{6}{|l|}{ PGRS $^{\mathrm{c}}$} \\
\hline Median & $-3.935 \times 10^{-3}$ & $-3.926 \times 10^{-3}$ & $-3.962 \times 10^{-3}$ & $-3.952 \times 10^{-3}$ & $7.238\left(8 \times 10^{-5}\right)$ \\
\hline Range & $\begin{array}{l}-4.397 \times 10^{-3} \text { to } \\
-3.509 \times 10^{-3}\end{array}$ & $\begin{array}{l}-4.45 \times 10^{-3} \text { to } \\
-3.386 \times 10^{-3}\end{array}$ & $\begin{array}{l}-4.363 \times 10^{-3} \text { to } \\
-3.463 \times 10^{-3}\end{array}$ & $\begin{array}{l}-4.427 \times 10^{-3} \text { to } \\
-3.422 \times 10^{-3}\end{array}$ & \\
\hline \multicolumn{6}{|l|}{$F_{\mathrm{SNP}}$} \\
\hline Median & $0.6 \times 10^{-3}$ & 0.001 & $-0.9 \times 10^{-3}$ & $0.7 \times 10^{-3}$ & $15.315\left(7 \times 10^{-10}\right)$ \\
\hline Range & -0.017 to 0.039 & -0.023 to 0.133 & -0.019 to 0.012 & -0.018 to 0.052 & \\
\hline \multicolumn{6}{|l|}{$F_{\text {miss }}$} \\
\hline Median & $0.5 \times 10^{-3}$ & $93 \times 10^{-6}$ & $0.1 \times 10^{-3}$ & $0.2 \times 10^{-3}$ & $74.885\left(<3 \times 10^{-16}\right)$ \\
\hline Range & $0.1 \times 10^{-3}$ to $5 \times 10^{-3}$ & $12 \times 10^{-6}$ to 0.01 & $\begin{array}{l}0.02 \times 10^{-3} \text { to } \\
8.9 \times 10^{-3}\end{array}$ & $\begin{array}{l}0.01 \times 10^{-3} \text { to } \\
12.8 \times 10^{-3}\end{array}$ & \\
\hline \multicolumn{6}{|l|}{$N_{\mathrm{ROH}}$} \\
\hline Median & 3 & 3 & 2 & 3 & $7.63\left(4 \times 10^{-5}\right)$ \\
\hline Range & $0-19$ & $0-40$ & $0-12$ & $0-21$ & \\
\hline \multicolumn{6}{|l|}{$F_{\mathrm{ROH}}$} \\
\hline Median & $1.652 \times 10^{-3}$ & $1.603 \times 10^{-3}$ & $1.331 \times 10^{-3}$ & $1.438 \times 10^{-3}$ & $1.659(0.174)$ \\
\hline Range & $0-41 \times 10^{-3}$ & $0-127 \times 10^{-3}$ & $0-12 \times 10^{-3}$ & $0-49 \times 10^{-3}$ & \\
\hline
\end{tabular}

${ }^{a}$ Age at Computerized Neurocognitive Battery test date

${ }^{\mathrm{b}}$ Values of estimated total intracranial volume were available only for a subset of participants $(n=385)$ with genetic and cognitive information included in the study (see Materials and methods-Image acquisition and pre-processing)

${ }^{c}$ Schizophrenia PGRS at the best-fitting $p_{\text {threshold }}(0.005)$, as mentioned in the manuscript

${ }^{\mathrm{d}}$ Between-group comparisons were performed using analysis of variance (ANOVA) tables for most of the variables displayed (continuous measures; $F$ and $p$-values reported), except for gender and medical status, where chi-squared tests were applied on the contingency tables

manuscripts [7, 8, 28], with slight modifications based on quality control procedures suggested elsewhere [29]. Briefly, each of the filtered genotyping batches was pruned for LD using VIF threshold $=10$, with window size $=50$ SNPs and window shift step $=5$ SNPs. Then, $F_{\mathrm{ROH}}$ was calculated with PLINK's sliding-window approach, with a minimum SNP length threshold and window size of 65 SNPs, $5 \%$ of missing SNPs allowed and no heterozygote SNPs accepted. The number of independent ROH segments was also extracted from the ".hom.indiv" files generated by PLINK, and is hereafter referred to as $N_{\mathrm{ROH}}$. Additional details on the ROHcalling algorithm are reported in Supplementary Material. 
Fig. 1 Distribution of age, age squared, total WRAT scores, and age-standardized WRAT scores. Notes: Age range in the uppermost left square is shown in years (8-22), and WRAT scores are displayed both before (raw) and after (std) age standardization. Numbers on the upper right triangle correspond to Pearson correlation coefficients between pairs of variables, and symbols indicate significance levels

$(\square<0.1, * p<0.05$, $* * p<0.01, * * * p<0.001)$

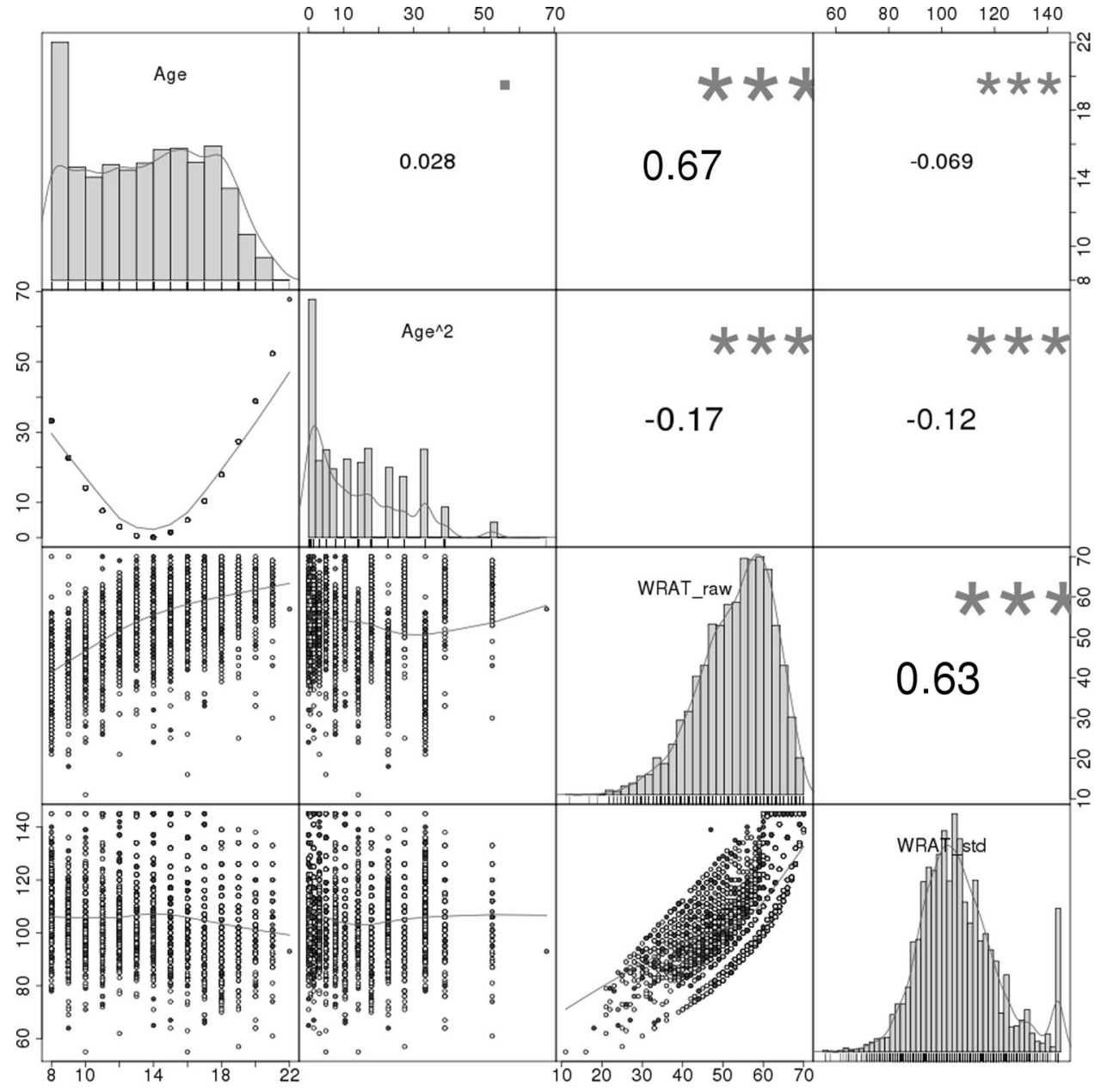

\section{Schizophrenia PGRS}

The imputed genotypes passing quality control (see Genotyping platforms and genotype imputation) were used to compute schizophrenia PGRS, based on data published by the Schizophrenia Working Group of the Psychiatric Genomics Consortium [30]. Initially, SNPs with ambiguous alleles (AT or CG), or in linkage disequilibrium with the local SNP with the smallest $p$-value were removed (the SNP with the smallest $p$-value within a $250 \mathrm{~kb}$ window is retained, and all neighbors with a linkage disequilibrium $r^{2}>0.1$ are removed; a step known as clumping). Also, SNPs within the major histocompatibility complex region (chromosome 6, 26-33 Mb) were omitted, and 500 different PGRS values were obtained ( $p$-value thresholds, $p_{\text {threshold, }}$, from 0.001 to 0.5 , with intervals of 0.001). The former procedures were implemented in PRSice.

As done in former studies [31-33], a specific $p$-value cutoff was chosen for the schizophrenia PGRS (out of the 500 estimations) by tuning the fitting parameters (adjusted $R^{2}$ ) to maximize the explained variance of independent regression models with cognitive performance as outcome and gender, age, batch, and PGRS as dependent variables.

\section{Image acquisition and pre-processing}

MRI was performed for a subset of participants at the Hospital of the University of Pennsylvania, by means of a 3T Siemens TIM Trio whole-body scanner (32-channel head coil; gradient performance: $45 \mathrm{mT} / \mathrm{m}$; maximum slew rate: $200 \mathrm{~T} / \mathrm{m} / \mathrm{s}$ ). The focus of the current study is on structural brain features derived from 3D T1-weighted images obtained using a MPRAGE sequence (TR: $1.81 \mathrm{~s}$; TE: $3.5 \mathrm{~ms}$; FA: $9^{\circ}$; FOV: $240 \times 180 \mathrm{~mm}$; $1 \mathrm{~mm}$ slice thickness; 160 slices), whose acquisition parameters have formerly been described in more detail [26].

Data from each participant were pre-processed using the recon-all stream from Freesurfer v5.3.0 (http://surfer.nmr. mgh.harvard.edu/) [34], using automatic parcellation and segmentation protocols to obtain 68 cortical and 14 subcortical gray matter brain regions, as well as some global brain features (e.g., intracranial volume (ICV)) [35]. Thickness and surface measurements from cortical regions 
were used for the ensuing analyses, along with volumetric estimates of subcortical volumes. Twelve relevant brain features were selected for analysis: intracranial and seven subcortical volumes used in a recent ENIGMA report [4], along with mean cortical thickness, total cortical surface area, cerebellar cortex volume, and cerebellar white matter volume. After merging with genetic and other phenotypic data of European-ancestry participants, there were 516 participants with MRI measures available. A measure of participants' age in months was extracted from the DICOM headers of the 516 MRI files, and it was used in the analyses involving brain features.

\section{Statistical analyses}

The analyses of ROH, PGRS, and cognition were performed via linear regression in $\mathrm{R}$, including sex, age, age squared, and ten genetic principal components as covariates. For the analysis of $\mathrm{ROH}$, fraction of missing values and SNP-by-SNP homozygosity were also adjusted for. Finally, the question of whether differences in brain features mediate the association between genomic features and IQ was assessed using causal mediation. Results reported below include $p$-values adjusted using either Bonferroni correction or false discovery rate $[36,37]$, which have been documented as appropriate methods to control for multiple testing. In the regression models, all continuous variables were scaled with R's "scale" function, to get interpretable coefficients. Additional details on the statistical methods are included in Supplementary Material.

\section{Results}

\section{Demographic and clinical data}

The data shown in Table 1 and Fig. 1 summarizes phenotypic information of PNC's sample subset included herein. When considering all participants, the correlations between age, age squared and total WRAT score were either small or moderate, with the largest coefficient for WRAT and age $(r=0.67)$. Even though the PNC participants were initially recruited randomly from the set of available genotypes regardless of genotyping platform [26], the age and IQ distributions displayed small yet statistically significant differences across batches (median ages: 13, 14, 15, and 16 years, $p<3 \times 10^{-16}$; median IQ scores: 105 and 106, $p=0.006$ ), which may have had an impact on the distribution of other variables such as ICV $(p=0.024$ for between-batch differences) and related neuroimaging measures. Besides, the contingency table of medical status categories (none/minor, mild, moderate, and severe) and genotyping platforms displayed unbalanced frequencies
( $p=10^{-10}$, Table 1$)$. These observations, along with the differences in genetic measures discussed below, encouraged the inclusion of genotyping platform in all ensuing analyses.

\section{Schizophrenia PGRS and cognitive performance}

As mentioned above (see Methods) 500 schizophrenia PGRS values were computed (to assess the $p$-value threshold $\left(p_{\text {threshold }}\right)$ range from 0.001 to 0.5 , in steps of 0.001). The model fitting parameters of PGRS for IQ are shown in Supplementary Fig. S1, using four different settings: models with or without participants displaying moderate/severe medical conditions, evaluated using either 4 or 15 covariates along with the PGRS. Outcomes were assessed based on both the overall adjusted- $R^{2}$ of the full model, and the PGRS's $p$-value within that model. Data on Supplementary Fig. S1 provide multiple insights: first, 15-covariate models showed higher adjusted- $R^{2}$ values than their 4-covariate counterparts, suggesting that the principal components extracted from the identity-by-state do explain part of the phenotypic variance in cognitive performance. Second, schizophrenia PGRS display smaller $p$-values when including participants with medical conditions. Third, across all models, the best-fitting parameters (smallest

$p$-values) throughout the different threshold values were observed using a $p_{\text {threshold }}$ of either 0.005 or 0.01 . Of note, when using standardized WRAT scores (a measure of IQ), 0.005 was also the threshold with the smallest $p$-values; it was thus selected for the analyses shown next (Supplementary Figs. S1, S2). The results indicate that a higher schizophrenia PGRS is associated with higher cognitive performance across age $(\beta=0.027, \mathrm{SE}=0.011$, $p=0.015$; Supplementary Fig. S3); $\beta$-values were positive across PGRS $p$-value thresholds in this model (range: $0.02-0.35$ ), as well as in all 4-covariate regressions and excluding participants with moderate/severe medical conditions. Lastly, there were no significant interactions between PGRS and either age or age squared on cognitive performance in the full dataset $(n=4183$; PGRS $\times$ age: $\beta=$ $-0.02, \mathrm{SE}=0.011, p=0.071 ; \mathrm{PGRS} \times \mathrm{age}^{2}: \quad \beta=0.014$, $\mathrm{SE}=0.011, p=0.21$ ), although there was an association with age when excluding participants with medical conditions $(n=3036$; PGRS $\times$ age: $\beta=-0.026, \mathrm{SE}=0.013$, $p=0.037$; PGRS $\times$ age $^{2}: \beta=0.019, \mathrm{SE}=0.013, p=0.139$; Supplementary Fig. S4). Those results were similar when using 4-covariate models.

\section{ROH and cognitive performance}

Descriptive values of $F_{\mathrm{ROH}}$ and related variables $\left(N_{\mathrm{ROH}}, F_{\mathrm{SNP}}\right.$, $\left.F_{\text {miss }}\right)$ are shown in Table 1 and Fig. 2. There were no 
Fig. 2 Correlation between different genomic features. Notes: F_SNP represents the SNP-by-SNP homozygosity; F_miss indicates the fraction of missing genotypes per sample; F_ROH is the fraction of the genome covered by homozygous blocks (included as variable of interest in the downstream analysis); N_ROH corresponds to the number of homozygous segments of each participant; PGRS_p0.005 denotes schizophrenia polygenic risk scores using markers with $p \leq 0.005$ in the PGC2 GWAS. Numbers on the upper right triangle correspond to Pearson correlation coefficients between pairs of variables, and symbols indicate significance levels $\left(\square p<0.1,{ }^{*} p<0.05,{ }^{* *} p<\right.$ $0.01, * * * p<0.001)$

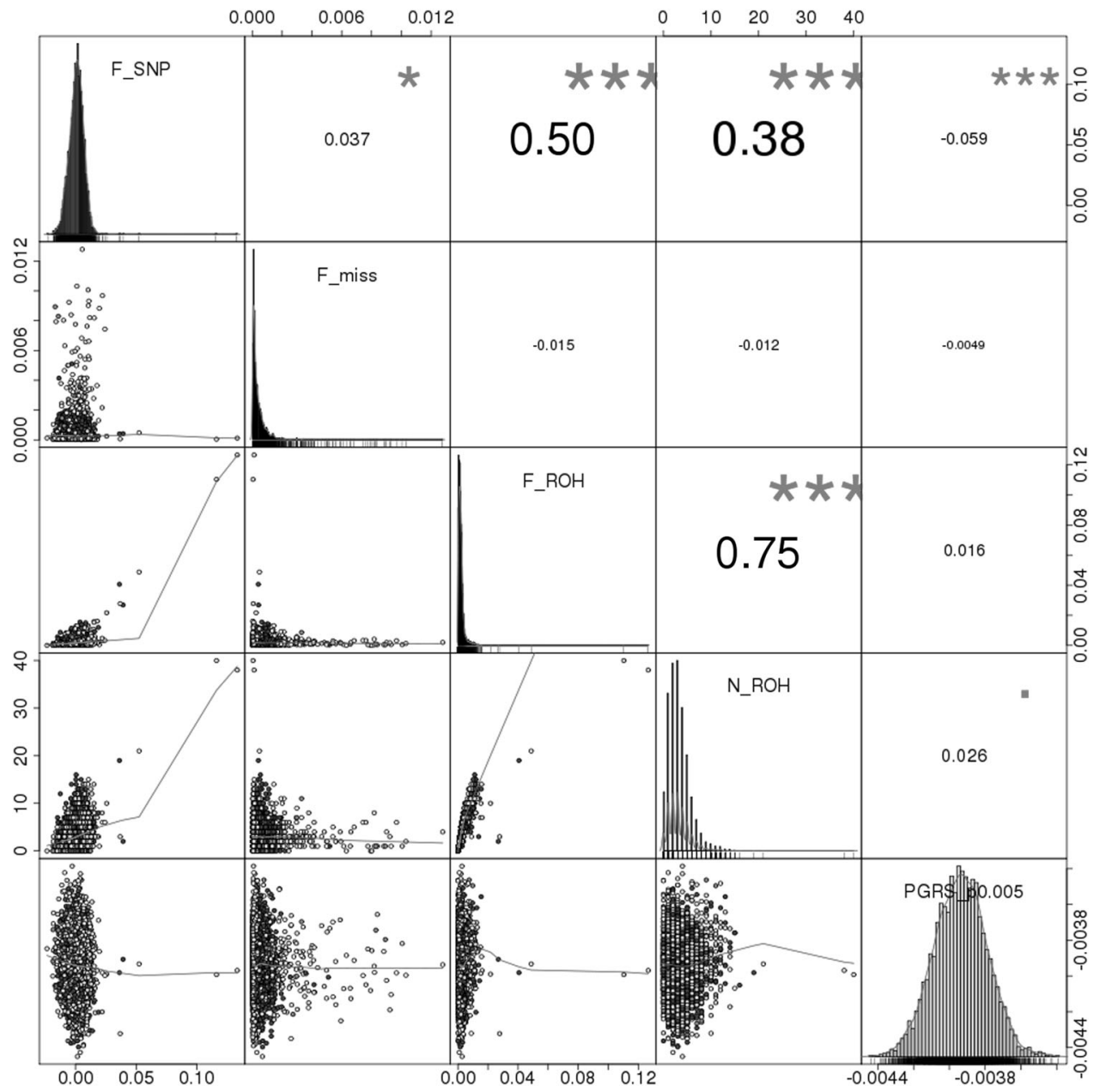

significant differences in the distribution of $F_{\mathrm{ROH}}$ across platforms, although a statistically significant batch-specific pattern was observed in $F_{\text {miss }}, F_{\mathrm{SNP}}$, and $N_{\mathrm{ROH}}\left(p<3 \times 10^{-16}\right.$, $p=7 \times 10^{-10}$, and $\left.p=4 \times 10^{-5}\right)$. Pairwise analysis of these variables indicated a moderate correlation between the number of $\mathrm{ROH}$ per individual $\left(N_{\mathrm{ROH}}\right)$ and SNP-by-SNP homozygosity $\left(F_{\mathrm{SNP}}\right)(r=0.38)$, whereas larger coefficients were observed between $F_{\mathrm{ROH}}$ and $F_{\mathrm{SNP}}(r=0.5)$, and between $F_{\mathrm{ROH}}$ and $N_{\mathrm{ROH}}(r=0.75)$. The latter is shown in more detail in Supplementary Fig. S5, where the $F_{\mathrm{ROH}}$ and $N_{\mathrm{ROH}}$ values are compared for the whole sample. The correlations remained virtually unchanged after removing participants with moderate/severe medical status. Those two distributions (with and without participants with relevant medical conditions) are highly overlapping, with only two out of six high-ROH burden participants $\left(F_{\mathrm{ROH}}>0.025\right)$ coming from the subsets of moderate and severe medical conditions. Of note, $F_{\mathrm{ROH}}$ was linearly independent of the schizophrenia PGRS (full sample: $r=0.016, p=0.293$; removing participants with medical conditions: $r=0.025, p=0.172$ ) (Fig. 2 and Supplementary Fig. S5), which further justifies the analysis of their joint effects in subsequent tests.
Regarding phenotype- $\mathrm{ROH}$ burden association tests, the output of linear regression analysis in the whole dataset $(n=$ 4183) showed a positive-signed association between cognitive performance and $F_{\mathrm{ROH}}\left(\beta=0.047, \mathrm{SE}=0.013, p=3.5 \times 10^{-}\right.$ ${ }^{4}$, Supplementary Fig. S6). Despite the apparent bias introduced by a few subjects with very high $F_{\mathrm{ROH}}$ values within the Quad platform, the effect was noticed across the four genotyping batches (Supplementary Fig. S7), and the analysis excluding subjects with $F_{\mathrm{ROH}}>0.02$ displayed similar outcomes $\left(\beta=0.057, \mathrm{SE}=0.012, p=1.3 \times 10^{-6}\right)$. The results after excluding participants with moderate/severe medical conditions (namely, keeping 3036 individuals) remained statistically significant $\left(\beta=0.046, \mathrm{SE}=0.014, p=1.6 \times 10^{-3}\right)$. An additional analysis revealed no interactions between $F_{\mathrm{ROH}}$ and either age or age squared on IQ $\left(F_{\mathrm{ROH}} \times\right.$ age: $\beta=0.011$, $\mathrm{SE}=0.011, \quad p=0.325 ; \quad F_{\mathrm{ROH}} \times \mathrm{age}^{2}: \quad \beta=-0.007, \quad \mathrm{SE}=$ 0.016, $p=0.672$; Supplementary Fig. S8).

\section{ROH $\times$ schizophrenia PGRS interaction on IQ}

A statistically significant interaction effect between $\mathrm{ROH}$ burden and schizophrenia PGRS on cognitive performance 


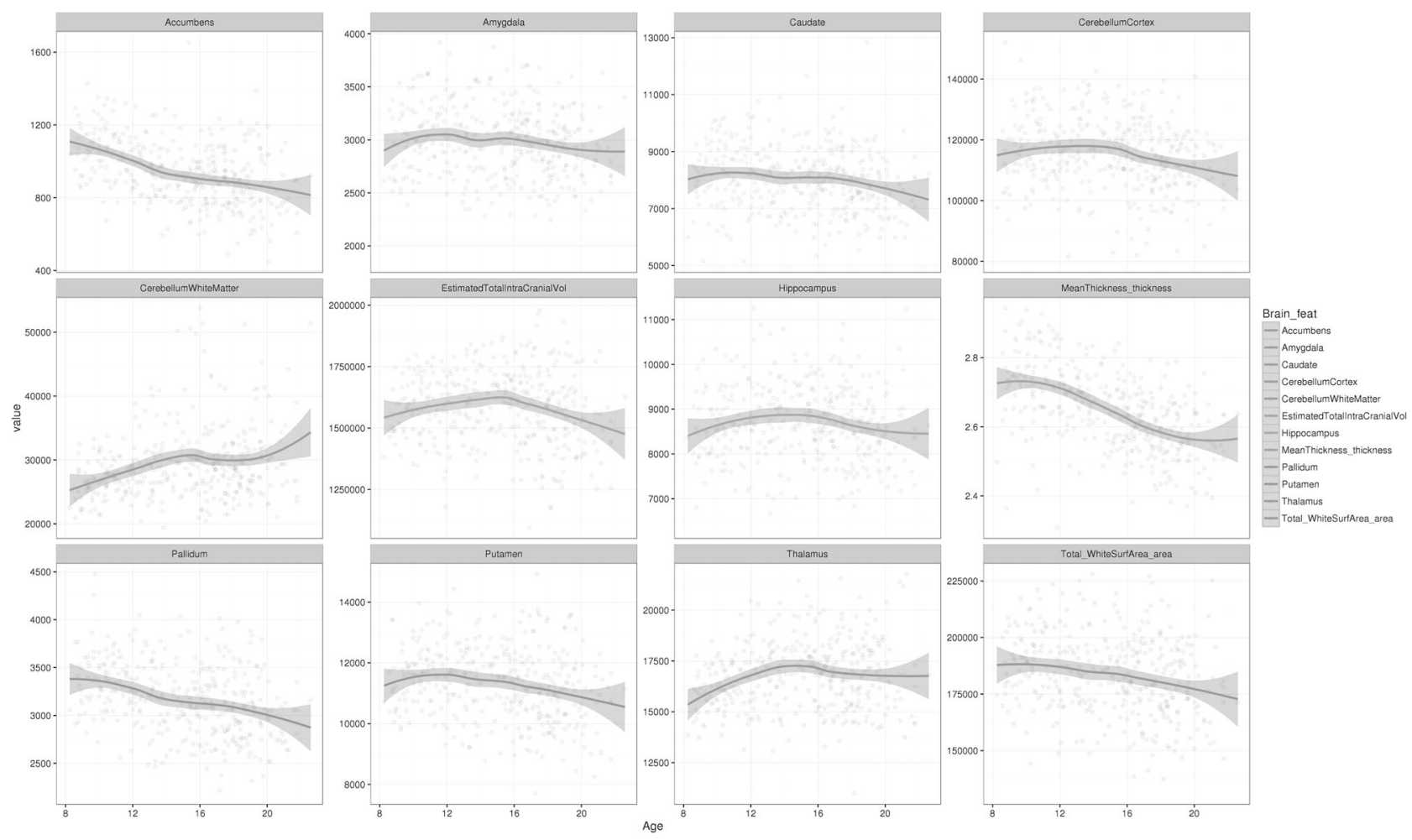

Fig. 3 Brain feature values, plotted against age. Notes: The vertical scales are in $\mathrm{mm}^{3}$ for most features (volumetric traits), except for mean cortical thickness (in $\mathrm{mm}$ ) and total cortical surface area (in $\mathrm{mm}^{2}$ ). Age (horizontal axis) is displayed in years

was detected, although the explained variance was relatively small. Briefly, the main effects model (15-covariate) showed significant results for both $F_{\mathrm{ROH}}(\beta=0.046, \mathrm{SE}=0.013$, $\left.p=5.2 \times 10^{-4}\right)$ and schizophrenia PGRS $(\beta=0.025, \mathrm{SE}=$ $0.011, p=0.023$ ), with an adjusted- $R^{2}$ of 0.4864 for the whole regression, whereas in the multiplicative interaction model $F_{\mathrm{ROH}} \times$ PGRS was statistically significant $(\beta=0.032$, $\mathrm{SE}=0.016, p=0.048$; overall adjusted- $\left.R^{2}: 0.4867\right)$. As indicated, there was slight increase in the model fitting parameter (adjusted- $R^{2}$ shifting from 0.4864 to 0.4867 ), with a statistically significant effect according to the ANOVA test for the interaction $(F=3.9, p=0.048)$. The results were very similar within the 4-covariate framework, with significant effects for both $F_{\mathrm{ROH}}$ and PGRS $\left(F_{\mathrm{ROH}}: \beta=0.037\right.$, $\mathrm{SE}=0.011, p=7.8 \times 10^{-4}$; PGRS: $\beta=0.027, \mathrm{SE}=0.011$, $p=0.015$; overall adjusted- $R^{2}: 0.4861$ ), with a significant interaction term and improved model-fitting statistics $\left(F_{\mathrm{ROH}} \times\right.$ PGRS: $\beta=0.036, \mathrm{SE}=0.016, p=0.029$; overall adjusted- $R^{2}: 0.4866$ ), and statistically significant ANOVA results $(F=4.8, p=0.029)$. The data in Supplementary Fig. S9 indicates that individuals with a high level of inbreeding would be more sensitive to the effects of schizophrenia polygenic burden: those in the uppermost $F_{\mathrm{ROH}}$ quartile would have higher cognitive performance when their schizophrenia PGRS is high. In contrast, subjects with lower $F_{\mathrm{ROH}}$ generally display no correlation between PGRS and cognition. As evidenced in Supplementary Fig. S9, despite statistically significant, the effect sizes of these interactions were relatively small. Of note, when excluding participants with high homozygosity burden $\left(F_{\mathrm{ROH}}>0.02\right)$, the main effects showed similar effect sizes and significance levels $\left(F_{\mathrm{ROH}}: \beta=0.055, \mathrm{SE}=0.012, p=2.5 \times 10^{-6}\right.$; schizophrenia PGRS: $\beta=0.024, \mathrm{SE}=0.011, p=0.032$ ), but the interaction term was not statistically significant $(\beta=0.009$, $\mathrm{SE}=0.011, p=0.422)$. There were no three-way interactions with age or age squared $\left(F_{\mathrm{ROH}} \times \mathrm{PGRS} \times\right.$ age: $\beta=-0.019, \mathrm{SE}=0.016, p=0.235 ; F_{\mathrm{ROH}} \times \mathrm{PGRS} \times \mathrm{age}^{2}:$ $\beta=-0.001, \mathrm{SE}=0.019, p=0.951)$. The significance of the findings remained unchanged after removing participants based on medical status.

\section{Brain features, ROH, and schizophrenia PGRS}

Descriptive values of the brain features, in relation to age and cognitive performance (age-standardized) are shown in Fig. 3 and Supplementary Fig. S10, respectively. Table 2 displays the results of 15-covariate models evaluating the associations either between brain features and $F_{\mathrm{ROH}}$ or between brain features and schizophrenia PGRS. After multiple testing adjustments, higher PGRS was significantly associated with smaller cortical surface area and smaller thalamic volume. Although there was no association 


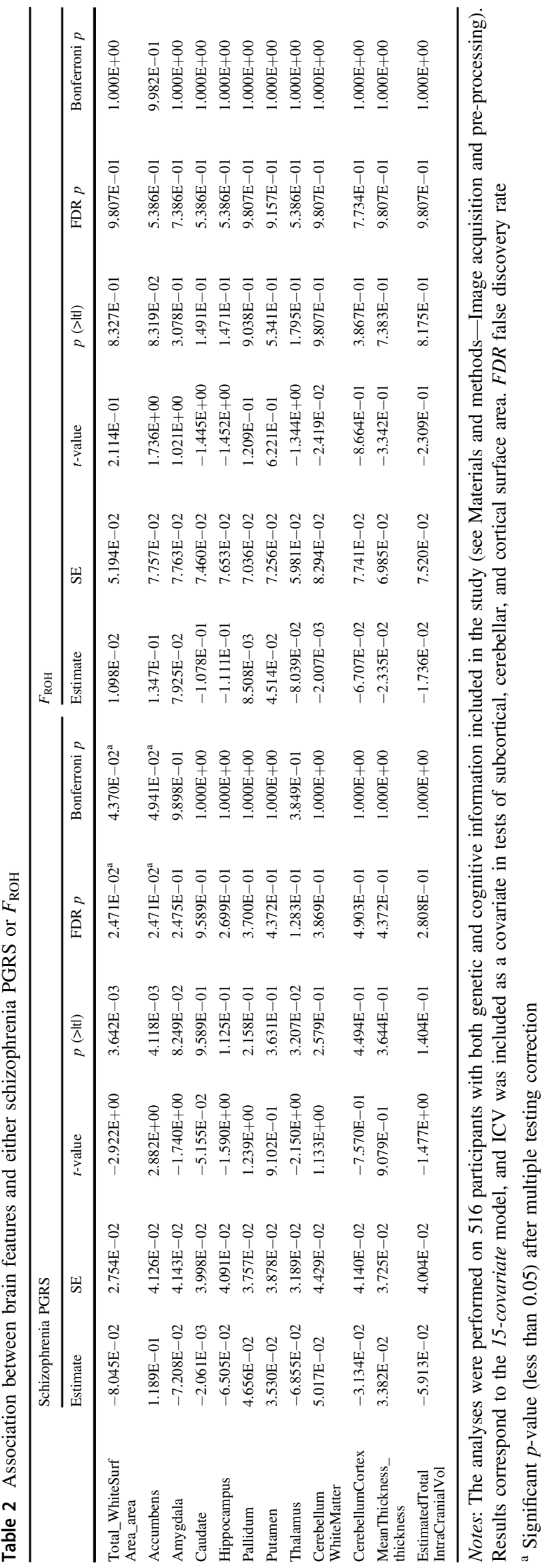

between PGRS and cognitive ability in the subset of participants with MRI data $(n=516)$, causal mediation analysis suggested a suppression effect: modifications of brain feature sizes would compensate against the direct effect of schizophrenia PGRS on cognitive ability (cortical area: average causal mediation effects (ACME) $=-0.026,95 \%$ C.I. $=[-0.059,-0.002], p=0.028$, average direct effect $(\mathrm{ADE})=0.058,95 \%$ C.I. $=[-0.115,0.232], p=0.52$; thalamus: $\quad \mathrm{ACME}=-0.021, \quad 95 \% \quad$ C.I. $=[-0.051$, $-0.0004], p=0.045, \mathrm{ADE}=0.053,95 \%$ C.I. $=[-0.119$, $0.225], p=0.55)$. Notice that such assertion had only limited support from the mentioned data subset, since no direct effects (ADE) of PGRS were detected.

With regard $F_{\mathrm{ROH}}$, there was no statistically significant association. PGRS $\times F_{\mathrm{ROH}}$ interaction tests did not reveal any significant association on the assessed brain features.

\section{Discussion}

In this work, the potential influence of both autozygosity and cumulative genetic risk for schizophrenia on cognitive performance was evaluated in a large and harmonized cohort $(n=4183)$ of European-ancestry participants from the general population, aged 8-22. Increased inbreeding, as indexed by a larger fraction of the genome in $\mathrm{ROH}$, was associated with higher cognitive performance. Similarly, a higher genetic burden for schizophrenia was related to higher cognitive scores. Although ostensibly paradoxical, the results agree with some previous reports using related study designs [7, 38, 39]. Additionally, interaction effects suggest that more inbred individuals might display higher cognitive test scores in the presence of high schizophrenia polygenic risk score. The relatively small effect sizes indicate that the contribution of these whole-genome features to the total heritability of cognitive performance is modest.

Regarding $\mathrm{ROH}$ and cognition, perhaps the most similar study to this one was conducted by Power et al. [7] in a demographically analog study sample (2329 Europeanancestry participants, age 12 ). The $\mathrm{ROH}$-calling procedures using both here and in the former report were also equivalent, and in both cases the outcomes suggest that, in young individuals, increased autozygosity is associated with higher intelligence. To interpret their findings, Power et al. [7] hypothesized that positive assortative mating in couples with better cognitive profiles could partly explain the mentioned association. Observations from other independent reports support the hypothesis of differential assortative mating patterns influencing cognitive, behavioral, and psychiatric phenotypes [9-14], which is possibly the most plausible framework to explain the findings from Power et al. [7] and the current report. Further research is needed since both research outcomes indicate relatively small effect 
sizes which might have been shadowed by unpublished findings.

Importantly, two former studies have found the inverse association in samples of adult participants. First, Joshi et al. [6] found a negative correlation between $\mathrm{ROH}$ and cognition by meta-analyzing information of 53,300 participants from different cohorts and ancestries. Importantly, as the same authors showed, both $F_{\mathrm{ROH}}$ and $N_{\mathrm{ROH}}$ vary depending on ancestry, which might have induced heterogeneity in that study that is not present in the current dataset. It is worth noting that Joshi et al. [6] included datasets (e.g., FTC_1) that are relatively similar to the present study sample (PNC) in terms of demographics, but there were no statistically significant results within those sub-cohorts. Differences in ROH-calling procedures could have also influenced this between-study discordance. Here, a validated protocol [28] of increasing popularity has been employed, which could strengthen the reliability of the findings. Another study by Howrigan et al. [8] showed, in a sample of 4854 European-ancestry adults from nine cohorts, that increased $\mathrm{ROH}$ burden might be associated with lower intelligence. The main focus on adult participants in both previous studies may limit the comparability of the results, since the genetics of intelligence exhibit largely dynamic patterns over the lifetime $[15,16]$.

Interestingly, we found a positive correlation between schizophrenia PGRS and cognitive performance, suggesting that higher genetic burden for schizophrenia is related to better cognitive performance. This finding somehow agrees with observations from two previous cohorts of healthy participants, which indicate that higher schizophrenia PGRS would be associated with decreased risk for psychosis-like experiences and schizotypy [38, 39]. The results from those two reports were interpreted by Barrantes-Vidal et al. [38] in view of potential involuntary biases in sampling: samples with only healthy participants are not likely to include subjects with high schizophrenia PGRS and high psychosisrelated phenotypes; people with high schizotypy and low resilience would seldom be part of the healthy general population, but would transition to clinical psychosis instead. Within that framework, lower genetic disease risk would protect healthy high-schizotypy individuals against transition to the clinical phenotype. A high-PGRS-lowresilience population subset would then rarely be part of a healthy sample, not because of an explicit sampling bias to exclude subclinical phenotypes, but rather due to psychopathological dynamics leading high-PGRS-low-resilience to the affected/patient groups. Of note, the current data (PNC) includes participants across a broad spectrum of phenotypes ranging from health to different diseases, and the association between higher schizophrenia PGRS and better cognition is more noticeable when removing participants with medical conditions. Namely, the positive correlation between PGRS and cognitive ability is stronger in the healthiest end of the phenotypic distribution, probably in concordance with an interplay between resilience and genetic schizophrenia risk as referred above.

Regarding the latter finding on schizophrenia PGRS and IQ, it is also important to notice that IQ has recently been reported to have a small negative genetic correlation with schizophrenia risk [40]. Those results obtained with LD Score Regression [41] are ostensibly in disagreement with the current findings, but it is also important to highlight that the data from the Brainstorm Consortium indicate a positive correlation between multiple psychiatric diseases (bipolar disorder, autism, and anorexia) and both education years and college attainment, and the absence of genetic correlation between cognitive performance and schizophrenia [42]. In addition, Okbay et al. [43] have also found a positive genetic correlation between years of education and schizophrenia; overall, there are mixed results that warrant further research to validate whether an increased genetic risk for schizophrenia is linked to the genetics of intelligence. While the arguments on unintended sampling bias outlined before-as opposed to true PGRS causalityare probably the most complete interpretation for the present findings, the latter evidences from other studies could also suggest that schizophrenia PGRS directly increases cognitive scores.

In addition to the explanation above, the observed association between increased genetic risk for schizophrenia and higher cognitive ability might partly be due to the link between psychosis PGRS and psychological traits such as creativity [44], which might be closely related to intelligence [45]. As discussed in the literature on psychometrics, there seem to be discontinuous populations when comparing IQ and creativity: creativity would be higher in individuals above a certain IQ score [45]; analogous non-steady relationships might also be postulated when stratifying IQ by genetic risk for psychosis: perhaps only above a given schizophrenia PGRS value, individuals would transition to psychosis and display cognitive alterations. Also, considering genetic evidence on schizophrenia as a by-product of human evolution [46, 47], one could postulate that the schizophrenia PGRS may confer some evolutionary advantages here manifested as increased cognitive performance.

It is worth noting that despite the important neuromaturational events occurring during adolescence that might affect schizophrenia liability (e.g., synaptic pruning and apoptosis) [48], the results here do not evidence age-related modulation of the PGRS-IQ and ROH-IQ associations. This ostensible independence of genetic effects on cognitive profiles seems to be stable across the considered age range, even when the age-cognition relationship clearly changes during adolescence (e.g., plateaus at $\sim 17$ years in Supplementary Figs. S4, S8). 
To our knowledge, this is the first systematic analysis of interactions between ROH and schizophrenia PGRS. The results show a nominally significant effect that deserves mention: schizophrenia PGRS would be positively correlated with IQ particularly in individuals with a high $\mathrm{ROH}$ burden. However, in view of the small size of the effects observed here, validation using larger independent samples is needed.

Limitations of the study include the medical conditions present in some participants, recruited from a hospital; however, as shown above, exclusion of individuals with moderate and severe medical status did not invalidate the findings. Moreover, the focus on a specific age range (8-22 years) and ancestry group has increased specificity, but the findings might not be generalizable to populations from different demographic and genetic backgrounds. Besides, the PGRS findings are nominally significant because of the model selection, and the parameters used to call $\mathrm{ROH}$ are relatively robust to confounding due to $\mathrm{CNVs}$ $[49,50]$, but one cannot completely rule out the possibility of artifacts in some of the measurements. Similarly, the procedure adopted here to tune PGRS $p$-value thresholds to find a best-fit model has been previously applied [31-33], but it renders significance of the main PGRS results only nominally significant in this case. Finally, the relatively small number of participants with MRI scans $(n=516)$ could have limited the power of brain-genetics tests considerably.

Acknowledgements Supported by the Research Council of Norway (204966, 249795, 223273, 213837); the South-Eastern Norway Regional Health Authority (2012-047, 2013-123, 2015-073, 2016083); European Community's 7th Framework Programme (602450, IMAGEMEND), and Kristian Gerhard Jebsen Foundation.

Author contributions AC-P and LTW conceived and designed the experiments, analyzed the data, and wrote the manuscript; AC-P, TK, DA, NTD, TM, and LTW performed the experiments, and all authors provided substantial input to and gave approval of the final version of the manuscript.

\section{Compliance with ethical standards}

Conflict of interest The authors declare that they have no conflict of interest.

\section{References}

1. Haworth CM, Wright MJ, Luciano M, et al. The heritability of general cognitive ability increases linearly from childhood to young adulthood. Mol Psychiatry. 2010;15:1112-20.

2. Schmitt JE, Neale MC, Fassassi B, et al. The dynamic role of genetics on cortical patterning during childhood and adolescence. Proc Natl Acad Sci USA. 2014;111:6774-9.

3. Paus T, Keshavan M, Giedd JN. Why do many psychiatric disorders emerge during adolescence? Nat Rev Neurosci. 2008;9:947-57.
4. Hibar DP, Stein JL, Renteria ME, et al. Common genetic variants influence human subcortical brain structures. Nature. 2015;520:224-9.

5. Alnæs D, Kaufmann T, Doan NT et al. Heritable cognitive and psychopathology factors in youth are predicted by brain fronto-temporal white matter pathway. JAMA Psychiatry. 2018;75:287-95.

6. Joshi PK, Esko T, Mattsson H, et al. Directional dominance on stature and cognition in diverse human populations. Nature. 2015;523:459-62.

7. Power RA, Nagoshi C, DeFries JC, Plomin R, Wellcome Trust Case Control C. : Genome-wide estimates of inbreeding in unrelated individuals and their association with cognitive ability. Eur J Hum Genet. 2014;22:386-90.

8. Howrigan DP, Simonson MA, Davies G, et al. Genome-wide autozygosity is associated with lower general cognitive ability. Mol Psychiatry. 2016;21:837-43.

9. Nordsletten AE, Larsson H, Crowley JJ, Almqvist C, Lichtenstein P, Mataix-Cols D. Patterns of nonrandom mating within and across 11 major psychiatric disorders. JAMA Psychiatry. 2016;73:354-61.

10. Peyrot WJ, Robinson MR, Penninx BW, Wray NR. Exploring boundaries for the genetic consequences of assortative mating for psychiatric traits. JAMA Psychiatry. 2016;73:1189-95.

11. Plomin R, Krapohl E, O'Reilly PF. Assortative mating-a missing piece in the jigsaw of psychiatric genetics. JAMA Psychiatry. 2016;73:323-4.

12. Robinson MR, Kleinman A, Graff M, et al. Genetic evidence of assortative mating in humans. Nat Human Behav. 2017;1:0016.

13. Mascie-Taylor CG. Spouse similarity for IQ and personality and convergence. Behav Genet. 1989;19:223-7.

14. Vandenburg SG. Assortative mating, or who marries whom? Behav Genet. 1972;2:127-57.

15. Deary IJ, Yang J, Davies G, et al. Genetic contributions to stability and change in intelligence from childhood to old age. Nature. 2012;482:212-5.

16. Trzaskowski M, Yang J, Visscher PM, Plomin R. DNA evidence for strong genetic stability and increasing heritability of intelligence from age 7 to 12. Mol Psychiatry. 2014;19:380-4.

17. Lencz T, Knowles E, Davies G, et al. Molecular genetic evidence for overlap between general cognitive ability and risk for schizophrenia: a report from the Cognitive Genomics consorTium (COGENT). Mol Psychiatry. 2014;19:168-74.

18. Hill WD, Davies G, Liewald DC, McIntosh AM, Deary IJ, Grp CCW. Age-dependent pleiotropy between general cognitive function and major psychiatric disorders. Biol Psychiat. 2016;80:266-73.

19. Germine L, Robinson EB, Smoller JW, et al. Association between polygenic risk for schizophrenia, neurocognition and social cognition across development. Transl Psychiat. 2016;6:e924.

20. Shafee R, Nanda P, Padmanabhan JL et al. Higher genetic risk of schizophrenia is associated with lower cognitive performance in healthy individuals. bioRxiv. 2017:103622. https://doi.org/10. 1101/103622.

21. Franke B, Stein JL, Ripke S, et al. Genetic influences on schizophrenia and subcortical brain volumes: large-scale proof of concept. Nat Neurosci. 2016;19:420-31.

22. Lee PH, Baker JT, Holmes AJ, et al. Partitioning heritability analysis reveals a shared genetic basis of brain anatomy and schizophrenia. Mol Psychiatry. 2016;21:1680-9.

23. Wilkinson GS, Robertson GJ, Psychological Assessment Resources Inc. WRAT 4: wide range achievement test; Professional manual. 4th ed. Lutz, FL: Psychological Assessment Resources, Inc.; 2006.

24. Gur RC, Richard J, Calkins ME, et al. Age group and sex differences in performance on a computerized neurocognitive battery in children age 8-21. Neuropsychology. 2012;26:251-65. 
25. Robinson EB, Kirby A, Ruparel K, et al. The genetic architecture of pediatric cognitive abilities in the Philadelphia Neurodevelopmental Cohort. Mol Psychiatry. 2015;20:454-8.

26. Satterthwaite TD, Elliott MA, Ruparel K, et al. Neuroimaging of the Philadelphia Neurodevelopmental Cohort. Neuroimage. 2014;86:544-53.

27. Vassos E, Di Forti M, Coleman J, et al. An examination of polygenic score risk prediction in individuals with first-episode psychosis. Biol Psychiatry. 2017;81:470-7.

28. Howrigan DP, Simonson MA, Keller MC. Detecting autozygosity through runs of homozygosity: a comparison of three autozygosity detection algorithms. BMC Genom. 2011;12:460.

29. Anderson CA, Pettersson FH, Clarke GM, Cardon LR, Morris AP, Zondervan KT. Data quality control in genetic case-control association studies. Nat Protoc. 2010;5:1564-73.

30. Schizophrenia Working Group of the Psychiatric Genomics C. Biological insights from 108 schizophrenia-associated genetic loci. Nature. 2014;511:421-7.

31. Kapoor M, Chou YL, Edenberg HJ, et al. Genome-wide polygenic scores for age at onset of alcohol dependence and association with alcohol-related measures. Transl Psychiatry. 2016;6:e761.

32. Maciejewski DF, Renteria ME, Abdellaoui A, et al. The association of genetic predisposition to depressive symptoms with non-suicidal and suicidal self-injuries. Behav Genet. 2017;47:3-10.

33. Tesli M, Espeseth T, Bettella F, et al. Polygenic risk score and the psychosis continuum model. Acta Psychiatr Scand. 2014;130:311-7.

34. Fischl B, Dale AM. Measuring the thickness of the human cerebral cortex from magnetic resonance images. Proc Natl Acad Sci USA. 2000;97:11050-5.

35. Desikan RS, Segonne F, Fischl B, et al. An automated labeling system for subdividing the human cerebral cortex on MRI scans into gyral based regions of interest. Neuroimage. 2006;31:968-80.

36. Benjamini Y, Hochberg Y. Controlling the false discovery rate: a practical and powerful approach to multiple testing. J R Stat Soc Series B Methodol. 1995;57:289-300.

37. Meyer-Lindenberg A, Nicodemus KK, Egan MF, Callicott JH, Mattay V, Weinberger DR. False positives in imaging genetics. Neuroimage. 2008;40:655-61.
38. Barrantes-Vidal N, Grant P, Kwapil TR. The role of schizotypy in the study of the etiology of schizophrenia spectrum disorders. Schizophr Bull. 2015;41:S408-16.

39. Zammit S, Hamshere M, Dwyer S, et al. A population-based study of genetic variation and psychotic experiences in adolescents. Schizophr Bull. 2014;40:1254-62.

40. Sniekers S, Stringer S, Watanabe K, et al. Genome-wide association meta-analysis of 78,308 individuals identifies new loci and genes influencing human intelligence. Nat Genet. 2017;49:1107-12.

41. Bulik-Sullivan B, Finucane HK, Anttila V, et al. An atlas of genetic correlations across human diseases and traits. Nat Genet. 2015;47:1236-41.

42. Anttila V, Bulik-Sullivan B, Finucane HK et al. Analysis of shared heritability in common disorders of the brain. bioRxiv. 2016:048991. https://doi.org/10.1101/048991.

43. Okbay A, Beauchamp JP, Fontana MA, et al. Genome-wide association study identifies 74 loci associated with educational attainment. Nature. 2016;533:539-42.

44. Power RA, Steinberg S, Bjornsdottir G, et al. Polygenic risk scores for schizophrenia and bipolar disorder predict creativity. Nat Neurosci. 2015;18:953-5.

45. Jauk E, Benedek M, Dunst B, Neubauer AC. The relationship between intelligence and creativity: new support for the threshold hypothesis by means of empirical breakpoint detection. Intelligence. 2013;41:212-21.

46. Srinivasan S, Bettella F, Mattingsdal M, et al. Genetic markers of human evolution are enriched in schizophrenia. Biol Psychiatry. 2016;80:284-92.

47. Banerjee $N$, Polushina $T$, Bettella $F$ et al. Human-specific methylated regions are enriched in schizophrenia. bioRxiv. 2017:113175. https://doi.org/10.1101/113175.

48. Cannon TD. How schizophrenia develops: cognitive and brain mechanisms underlying onset of psychosis. Trends Cogn Sci. 2015;19:744-56.

49. Nalls MA, Guerreiro RJ, Simon-Sanchez J, et al. Extended tracts of homozygosity identify novel candidate genes associated with late-onset Alzheimer's disease. Neurogenetics. 2009;10:183-90.

50. Nalls MA, Simon-Sanchez J, Gibbs JR, et al. Measures of autozygosity in decline: globalization, urbanization, and its implications for medical genetics. PLoS Genet. 2009;5:e1000415. 